\title{
Cortical Thinning Correlates with Cognitive Change in Multiple Sclerosis but not in Neuromyelitis Optica
}

Yaou Liu, ${ }^{1, \#}$, Teng Xie ${ }^{3 \#}$, Yong He ${ }^{3}$, Yunyun Duan ${ }^{1}$, Jing Huang ${ }^{1}$, Zhuoqiong Ren ${ }^{1}$, Gaolang Gong ${ }^{3}$, Jun Wang ${ }^{3}$, Jing Ye ${ }^{4}$, Huiqing Dong ${ }^{4}$, Helmut Butzkueven ${ }^{5}$, Fu-Dong Shi ${ }^{2}$, Ni Shu ${ }^{3}$, Kuncheng $\mathrm{Li}^{1 *}$

${ }^{1}$ Department of Radiology, Xuanwu Hospital, Capital Medical University, Beijing 100053, P. R. China

${ }^{2}$ Department of Neurology and Tianjin Neurological Institute, Tianjin Medical University General Hospital, Tianjin, 300052, P. R. China

${ }^{3}$ State Key Laboratory of Cognitive Neuroscience and Learning, Beijing Normal University, Beijing 100875, P. R. China

${ }^{4}$ Department of Neurology, Xuanwu Hospital, Capital Medical University, Beijing 100053, P. R. China

${ }^{5}$ Department of Medicine, University of Melbourne, Parkville 3010, Australia

\# Yaou Liu and Teng Xie contributed equally to this work

\section{${ }^{\star}$ Corresponding Authors:}

Kuncheng Li, MD, Department of Radiology, Xuanwu Hospital, Capital Medical University, Beijing 100053, China. Tel: +86 13911099059; Fax: +86 1083198376 . E-mail: kunchengli55@gmail.com 



\title{
Cortical Thinning Correlates with Cognitive Change in Multiple Sclerosis but not in Neuromyelitis Optica
}

\begin{abstract}
Objectives: To compare spatial patterns of cortical thickness alterations in neuromyelitis optica (NMO) and multiple sclerosis (MS); and to investigate the correlations between cortical thinning and clinical variables in NMO and MS.

Methods: We studied 23 patients with NMO, 27 patients with MS and 26 healthy controls (HC). The global, brain region and vertex-based cortical thickness (CTh) were analysed and compared among the three groups. A general linear model was used to investigate the correlations between cortical thinning and clinical measures.

Results: A limited number of cortical regions in visual cortex were found to be significantly thinner in NMO patients than in HC. The MS patients exhibited more widespread cortical thinning compared with $\mathrm{HC}$, and significantly greater cortical thinning in the insula and the parahippocampus compared with NMO. The extent of cortical thinning in several brain regions correlated with cognitive measures in MS, but not in NMO.

Conclusions: Neocortical thinning in NMO mainly affects visual cortex, while MS patients show much more extensive cortical thinning. Cognitive changes are correlated with cortical atrophy in MS not in NMO. The substrates of cognitive changes in MS and NMO could therefore be different.
\end{abstract}

Key Words: multiple sclerosis; neuromyelitis optica; cortical thickness; cognitive impairment

Key Point1: MS patients show much more extensive cortical thinning than NMO.

Key Point 2: Cortical thinning of insula and parahippocampus particularly distinguishes MS from NMO.

Key Point 3: Cognitive changes are correlated with cortical atrophy in MS not in NMO. 


\section{Introduction}

Neuromyelitis optica (Devic's disease) (NMO) is an inflammatory astrocytopathy and demyelinating disease of the central nervous system (CNS) that is characterized by severe attacks of the optic neuritis and myelitis, often without significant cerebral pathology seen on T2 or FLAIR sequences [1; 2]. Brain abnormalities in NMO were shown in several studies using different MRI modalities [3-8].Simple white matter diffusion measures of NMO patients, such as mean diffusivity histograms were reported as normal[5] or showed abnormalities in motor and visual systems only[9]. However, more advanced diffusion techniques reported significant changes in brain WM in NMO, for example using tract-based spatial statistics[6],or small world network properties[10]. Whether brain atrophy occurs in NMO is controversial. Mild grey matter atrophy in frontal, temporal and insular regions was detected in by voxel-based morphology (VBM) in one previous study[11], but not confirmed in others [12; 13].

Cortical thickness is a more reliable, direct and biologically significant measurement of atrophy than cortical volume due to the low variability in the cytoarchitectural structure of the grey matter[14; 15$]$.Measurements of cortical thickness can reflect the size, density and arrangement of cells. Measuring cortical thickness provides important information regarding regional integrity of the cerebral cortex, thus yielding new insights into disease pathology[16-19].Currently, there is only one study[20] that reported mild cortical thinning in the visual and sensorimotor cortex in NMO patients.

Cognitive impairment is a common co-morbidity of MS which detrimentally affect many aspects of daily life, with prevalence rates ranging from $43 \%$ to $70 \%$, and cortical atrophy correlates with cognitive defects in MS[21]. In NMO, cognitive change has been reported [7], but the relationship between cortical atrophy and cognitive impairment in NMO has not previously been assessed. In the present study, we analysed global and regional cortical 
thickness (CTh) in NMO patients, and directly compared the findings with a cohort of MS patients and healthy controls $(\mathrm{HC})$, using high-resolution isotropic 3.0T MRI data. Furthermore, we investigated the correlations between the identified cortical atrophy and clinical characteristics including cognitive scores in both diseases.

\section{Materials and Methods}

\section{Participants}

The institutional review board of Xuanwu Hospital approved the study and written informed consent was obtained from each participant. We consecutively recruited 23 NMO patients (3males, 20females; mean age 35.5years, SD 10.8 years), and 27 relapsing-remitting MS (RRMS)patients (8 males, 19 females; mean age 33.2 years, SD 9.4 years) from the Department of Neurology in Xuanwu Hospital, Capital Medical University from January 2012 to September 2013. The RRMS diagnosis was made according to the modified McDonald criteria [22] and NMO diagnosis was determined according to the revised diagnostic criteria (absolute criteria: optic neuritis and myelitis, the presence of at least two of the following three additional criteria: (1) brain MRI results negative or non-diagnostic for MS at onset, (2) MRI evidence of a spinal cord T2 lesion of three or more vertebral segments, and (3) a serological test result positive for NMO antibodies) [2]. All of the NMO patients were relapsing NMO and eighteen NMO patients (78.3\%) were AQP4 antibody positive. Eighteen of the NMO patients had no white matter FLAIR or T2 hyperintensities identified by cerebral MRI and five had non-specific T2/FLAIR white matter lesions. None of the participating patients had been treated with disease-modifying medications (e.g., corticosteroids and immunosuppressants) in the 2 weeks before the MR images were obtained. The main demographic and clinical characteristics including mini-mental state examination (MMSE), paced auditory serial addition test 3 seconds version (PASAT3), paced auditory serial addition test 2(PASAT2), Expanded Disability Status Scale (EDSS), and disease duration of the patients studied are reported in Table 1. We chose 26 sex- and age-appropriate healthy controls (HC) $(9$ males, 17 females; mean age 35.1 years, SD 11.3 years) with no previous history of 
neurological dysfunction and with normal findings on neurological examination and MRI (Table 1).

\section{Image Acquisition}

Imaging was performed on a 3.0T MR system (Trio Tim Siemens, Germany) in the Department of Radiology, Xuanwu Hospital, Capital Medical University. A standard head coil was used with foam padding to restrict head motion. The routine axial slices were positioned to run parallel to a line that joins the AC-PC line of the corpus callosum, with an identical field of view $(256 \mathrm{~mm} \times 256 \mathrm{~mm})$, number of sections $(35)$, section thickness $(4 \mathrm{~mm})$. (a) Axial T2-weighted turbo spin echo (repetition time $[\mathrm{TR}]=5000 \mathrm{~ms}$, echo time $[\mathrm{TE}]=$ $87 \mathrm{~ms}$, number of signals acquired $=1$, echo train length $=15$, matrix size $=256 \times 256$ ), (b) Axial Fluid-attenuated inversion recovery (FLAIR) sequence (TR/TE=8500/87ms, inversion time $[\mathrm{Tl}]=2500 \mathrm{~ms}$, number of signals acquired=1, matrix size=256 $\times 256$ ), and (c) Sagittal three-dimensional (3D)Volumetric T1-weighted magnetization-prepared rapid acquisition gradient echo(MPRAGE) (TR/TE $=1600 / 2.13 \mathrm{~ms}, \mathrm{Tl}=1000 \mathrm{~ms}$, flip angle $=9^{\circ}$, $\mathrm{FOV}=256 \mathrm{~mm} \times 224 \mathrm{~mm}$, matrix size $=256 \times 224$, slice thickness $=1.0 \mathrm{~mm}$, voxel dimensions $=1.0 \mathrm{~mm} \times 1.0 \mathrm{~mm} \times 1.0 \mathrm{~mm})$ images were obtained. The routine sequences $(\mathrm{T} 2 \mathrm{WI}$ and FLAIR) were used to identify brain lesions and 3D MPRAGE was acquired for cortical thickness measurement.

\section{Cortical Thickness Measurement}

We utilized a routine pipeline of the CIVET software (version 1.1.9, Montreal Neurological Institute at McGill University, Montreal, Quebec, Canada) to extract cortical thickness from the structural MRI images. The flowchart of the software to evaluate the cortical thickness was shown in Figure 1. Briefly, the original images were first registered to stereotaxic space with linear transformation while the non-uniformity artefacts were corrected using the N3 algorithm[23]. Then the registered and corrected images were automatically segmented into grey matter, white matter, cerebro-spinal fluid and background using an advanced neural net classifier [24]. The inner and outer grey matter surfaces, with a total 
of 81,924 polygons, $(40,962$ vertices each hemisphere) were then automatically extracted from each magnetic resonance (MR) volume using the constrained Laplacian-based automated segmentation with proximities (CLASP) algorithm [25]. Cortical thickness (CTh) was thus defined as the distance between linked vertices on the inner and outer surfaces[26]. Finally, a $20 \mathrm{~mm}$ smoothing was applied to improve sensitivity [27]. The cortical thickness measurement approaches have previously been validated with high sensitivity and reproducibility using both manual measurements [28] and simulation approaches [26]. Furthermore, this method has been applied to study neurological diseases such as multiple sclerosis [29], Alzheimer's disease [14; 30]and schizophrenia [31].

\section{Statistical Analysis}

An analysis of covariate (ANCOVA) was employed to explore the CTh differences among the three groups. Age, gender and years of education were taken as covariates. We tested the mean CTh measurements of the whole brain, each of the hemispheres, all the 78 regions of interests from the surfaced-based Automated Anatomical Labeling (AAL) template, and CTh on each vertex. The significance level for lobar CTh, AAL CTh and vertex-based comparisons was determined as $p<0.05$ after false discovery rate (FDR) correction. Furthermore, we employed the areas presenting significant main effect of group in CTh ANCOVA as a mask to test the correlation between cognitive scores and CTh at all the scales mentioned above. To do this, we constructed a general linear model, selecting CTh as the dependent variable, and clinical scores including cognitive scores as the independent variable and age, gender and years of education as covariates. All the vertex-based results were visualized using BrainNet Viewer software (version 1.4, http://www.nitrc.org/projects/bnv/).

\section{Results}

\section{Demographic and Neurological Evaluations}

As shown in Table 1, no significant differences were observed in age, gender and years of 
education among the 3 groups (RRMS, NMO and $\mathrm{HC}$ ). The NMO patients showed higher EDSS than MS patients. The disease duration, PASAT3, PASAT2 and MMSE showed no significant difference between MS and NMO subjects $(p=0.45,0.63,0.48$ and 0.79 respectively). However, both patient groups revealed significantly lower scores of PASAT3, PASAT2 and MMSE compared to the HC group.

\section{Global, Hemispheric, Lobe and Brain Regional Cortical Thickness Differences}

The mean CTh was significantly different among the three groups (MS, NMO, HC) $(p=0.0022)$. The MS patients had significantly thinner cortices (global measure) than NMO patients $(p=0.046)$ and $\mathrm{HC}(p=0.0005)$. Individual hemispheres showed similar results (left hemisphere: F-test $p=0.0021$, MS versus $H C p=0.0005$, MS versus NMO $p=0.05$; right hemisphere: F-test $p=0.0013$, MS versus $H C p=0.0003$, MS versus NMO $p=0.033$ )(Figure 2). Compared to $\mathrm{HC}$, the MS patients had widely distributed CTh thinning in bilateral temporal and occipital lobes, the left frontal lobe and the right parietal lobe, and in the right temporal lobe compared with the NMO group ( $p<0.05$ FDR corrected, Table 2$)$. The NMO group was no different to $\mathrm{HC}$ at the global, hemispheric or lobar level, but a trend towards thinner cortex measures was observed in the left occipital lobe $(p=0.023$ uncorrected, $\mathrm{p}=0.18$ FDR corrected). The analysis of AAL regional cortical thinning showed reduced CTh in brain regions located in temporal, occipital, frontal and parietal lobes in MS patients compared to HC. Several regions also showed more severe thinning in MS compared to NMO, limited to the temporal and occipital lobes, while significant CTh thinning was only found in the left calcarine fissure and surrounding cortex (within left visual cortex) in comparisons of NMO patients with HC ( $p<0.05$ FDR corrected, Table 3$)$.

\section{Vertex-wise Cortical Thickness Differences}

The vertex-wise ANCOVA revealed results in accordance with the above results at brain lobe and brain region levels. It is evident that the areas showing a significant difference in CTh among the 3 groups $(p<0.05$, FDR corrected) were widely distributed in the cerebral 
cortex (Figure 3A). MS patients had thinner cortices than the HC group in the bilateral occipital, medial temporal, left inferior frontal and the right parietal brain regions (Figure 3B). The MS group also showed significant cortical thinning, compared to the NMO group, in the left parahippocampus, bilateral insula, and the left inferior occipital gyrus (Figure 3C). The cortical thickness reduction found in the NMO group compared to the HC group was limited to the left middle occipital gyrus (Figure 3D).

\section{Association between CTh Changes and Clinical Tests Including Cognitive Scores in Patients with NMO and MS}

In MS patients, significant correlations between atrophy of the left parahippocampus, left fusiform and left superior frontal orbital gyri and both PASAT3 and PASAT2 $(p<0.01$, corrected for age, sex and educational status) were shown at vertex-level (Figure 4A,B). No correlation with the cognitive changes was found at whole brain, lobe or AAL brain region level. A significant negative correlation between the disease duration and cortical atrophy was found in six brain regions (right calcarine, right Heschl'sgyrus, right parahippocampal gyrus, right lingual gyrus, right insula and left anterior cingulate gyrus, all $p<0.01$ ) at the AAL brain region level and confirmed at the vertex level (Figure $4 \mathrm{C}$ ). No significant correlation was identified between EDSS and cortical thinning in MS.

In NMO patients, no significant correlations were observed between cortical atrophy and disease duration, EDSS or cognitive test at either AAL brain region or vertex level.

\section{Discussion}

In this study, we investigated global and regional grey matter changes in relatively well matched NMO and MS patients and healthy controls by measuring CTh. We measured the CTh at five size levels: global mean CTh, average hemisphere CTh, average lobe CTh, brain regional CTh, and vertex-wise CTh. Compared with the controls, no significant cortical thinning was observed in the NMO patients at global, hemispherical, and lobar level. However, at the brain region and vertex-wise level, a limited number of cortical 
regions in visual cortex were found to be significantly thinner in NMO patients than $\mathrm{HC}$. These findings are consistent with previous studies of cortical volume loss in NMO using either the VBM method [11] or the cortical measurement method using Freesurfer software [20; 32]. Visual cortex thinning in NMO is most likely secondary to the severe optic nerve involvement in this disease. A recent study from von Glehn and colleagues[32] found a correlation between retinal nerve fibre layer (RNFL) thinning and pericalcarine cortical thickness, which supports the hypothesis of retrograde and anterograde neurodegeneration as the cause of GM atrophy in NMO.

In contrast, the MS patients exhibited widespread cortical thinning compared with HC. This is consistent with previous studies using either cortical thickness $[17 ; 18]$ as a measurement or the VBM method [11; 33] of assessing cortical volume loss. The current study extends previous studies by demonstrating the spatial patterns of cortical thinning in all three groups using the same software and imaging equipment

Two recent neuropathological studies revealed different pathological changes in NMO cerebral cortex. One study revealed an absence of cortical demyelinating lesions, but widespread astrogliosis and neuronal pathology in cerebral cortex[8], while the other showed both inflammatory demyelinating events characterized by pattern-specific loss of AQP4 immune activity and cortical neurodegeneration in $\mathrm{NMO}$ [34]. While these might seem discordant with our results and some other previous MRI findings in assessing brain atrophy in NMO [11;20], MRI examinations are typically performed in patients with mild to modest disease burden and pathological studies are usually conducted in end-stage disease, assessing long-term pathological outcomes. Alternatively, current MRI techniques may be not sensitive enough to detect all the pathological findings.

Interestingly, subtle widespread white matter damage or atrophy was observed in several previous studies in NMO [6; 13; 35], contrasting with the very limited cortical thinning shown in our study, which could argue for predominant white matter involvement in NMO. 
Among many possible explanations for differential involvement of grey and white matter in $\mathrm{NMO}$, expression of different isoforms of $\mathrm{AQP}$, or differential blood brain barrier (BBB) permeability between grey and white matter are very plausible [36; 37].

The MS patients showed reduced global cortical thickness and widespread cortical thinning in many brain regions involving all the lobes, bilaterally. The pathological factors determining cortical thinning include cortical demyelination, neuronal loss, or secondary degeneration from white matter lesions[38].Compared with the NMO patients, cortical atrophy in MS was much more widespread and severe, showing a distinct pattern of cortical thickness changes. Interestingly, the largest differences between CTh in MS and $\mathrm{NMO}$ were in the insula and the parahippocampus, confirming results on one previous comparative study using VBM[11]. Furthermore, a previous study also showed that highly significant focal atrophy in temporal cortex occurred early in the MS disease course [18], and early insular and parahippocampus atrophy could therefore be a key feature distinguishing MS and NMO.

Interestingly, significantly reduced cognitive performance including MMSE, PASAT2, PASAT3 was found in NMO patients, of a similar magnitude to MS patients, matched for age, disease duration and educational level. This cognitive change was correlated with areas of reduced cortical thickness in MS patients, in the left parahippocampus, left fusiform and left superior frontal orbital gyri. No such associations were found in NMO. Our study therefore suggests that the pathological substrate of impaired cognition in MS and NMO could be different; specifically that cognitive change is linked to cortical atrophy in MS but not in NMO. We believe that altered cognition in NMO may be correlated to widespread subtle abnormalities in white matter as reported by us and others $[6 ; 13 ; 35]$, and/or to similar subtle abnormalities in grey matter, for instance abnormalities on magnetic transfer ratio and diffusion histogram derived metrics[5]. Larger, longitudinal studies correlating cognitive change with advanced MRI measures will be required to better understand the evolution of relevant clinico-pathological correlations and identify 
the MRI biomarkers for monitoring cognitive impairment in MS and NMO. On the other hand, our study provides further evidence that cognitive change in MS is linked to cortical atrophy[39; 40], especially frontal and temporary atrophy, and that medical intervention reducing atrophy progression could reduce cognitive change, a concept that will need to be proven in large prospective studies.

\section{Limitations}

The present study had several methodological limitations. First of all, the sample size was relatively small. The significance and reliability of the results would be improved by enrolling more subjects, and compare the NMO patients in different subgroups: with brain lesions vs without brain lesions; NMO-IgG positive vs negative; or cognitive impairment vs cognitive preserved. Secondly, subcortical grey matter regions are structurally different from cortical regions, they do not have an inner surface of grey matter, so the estimation of thickness by the method we used is not applicable to subcortical grey matter regions such as the hippocampus, thalamus and caudate, which are known to be affected early in MS. Finally, the cognitive assessment of the participants in the current study only includes MMSE and PASAT, and a more systematical cognitive evaluation, for example using Minimal Assessment of Cognitive Function in MS (MACFIMS), will be performed in a planned future study.

In conclusion, limited cortical thinning in NMO was found mainly in the visual cortex, while MS patients showed much more extensive cortical thinning. The main difference of cortical atrophy between MS and NMO was located in insula and parahippocampus, which could potentially help distinguish these two diseases. Cognitive changes are correlated with cortical atrophy in MS not in NMO, implying the substrates of cognitive changes in MS and NMO may be different.

\section{Acknowledgements}

We would like to thank Dr Alan Evans for kindly providing the MNI CIVET software. 
The scientific guarantor of this publication is Kuncheng Li. The authors of this manuscript declare no relationships with any companies, whose products or services may be related to the subject matter of the article. This study has received funding by the McDonald Fellowship from Multiple Sclerosis International Federation (YL), 973 Program (2013CB837300), the National Science Foundation of China (Nos. 81101038, 30930029, 30800267, 81000633 and 81030028), the National Science Fund for Distinguished Young Scholars (No. 81225012), the Beijing Natural Science fund (No.7133244), the Beijing Funding for Training Talents (YH) and Major Project of National Social Science Foundation (No. 11\&ZD186). Dr Ni Shu and Dr. Teng Xie have significant statistical expertise. Institutional Review Board approval was obtained. Written informed consent was obtained from all subjects (patients) in this study. Methodology: prospective, cross sectional study, performed at one institution.

\section{References}

1 Wingerchuk DM, Lennon VA, Lucchinetti CF, Pittock SJ, Weinshenker BG (2007) The spectrum of neuromyelitis optica. Lancet Neurol 6:805-815

2 Wingerchuk DM, Lennon VA, Pittock SJ, Lucchinetti CF, Weinshenker BG (2006) Revised diagnostic criteria for neuromyelitis optica. Neurology 66:1485-1489

3 Pittock SJ, Lennon VA, Krecke K, Wingerchuk DM, Lucchinetti CF, Weinshenker BG (2006) Brain abnormalities in neuromyelitis optica. Arch Neurol 63:390-396

4 Rocca MA, Agosta F, Mezzapesa DM et al (2004) A functional MRI study of movement-associated cortical changes in patients with Devic's neuromyelitis optica. Neuroimage 21:1061-1068

5 Rocca MA, Agosta F, Mezzapesa DM et al (2004) Magnetization transfer and diffusion tensor MRI show gray matter damage in neuromyelitis optica. Neurology 62:476-478

6 Liu Y, Duan Y, He Y et al (2012) A tract-based diffusion study of cerebral white matter in neuromyelitis optica reveals widespread pathological alterations. Mult Scler 18:1013-1021

7 Blanc F, Zephir H, Lebrun C et al (2008) Cognitive functions in neuromyelitis optica. Arch Neurol 65:84-88

8 Popescu BF, Parisi JE, Cabrera-Gomez JA et al (2010) Absence of cortical demyelination in neuromyelitis optica. Neurology 75:2103-2109

9 Yu C, Lin F, Li K et al (2008) Pathogenesis of normal-appearing white matter damage in neuromyelitis optica: diffusion-tensor MR imaging. Radiology 246:222-228 Liu Y, Duan Y, He Y et al (2012) Altered topological organization of white matter structural networks in patients with neuromyelitis optica. PLoS One 7:e48846

11 Duan Y, Liu Y, Liang P et al (2012) Comparison of grey matter atrophy between patients with neuromyelitis optica and multiple sclerosis: A voxel-based morphometry study. Eur J Radiol 81:e110-114

12 Blanc F, Noblet V, Jung B et al (2012) White matter atrophy and cognitive dysfunctions in neuromyelitis optica. PLoS One 7:e33878

13 Chanson JB, Lamy J, Rousseau F et al White matter volume is decreased in the brain of patients with neuromyelitis optica. Eur J Neurol

14 Lerch JP, Pruessner JC, Zijdenbos A, Hampel H, Teipel SJ, Evans AC (2005) Focal decline of 
cortical thickness in Alzheimer's disease identified by computational neuroanatomy. Cereb Cortex 15:995-1001

Fischl B, Dale AM (2000) Measuring the thickness of the human cerebral cortex from magnetic resonance images. Proc Natl Acad Sci U S A 97:11050-11055

Calabrese M, Agosta F, Rinaldi F et al (2009) Cortical lesions and atrophy associated with cognitive impairment in relapsing-remitting multiple sclerosis. Arch Neurol 66:1144-1150 Calabrese M, Rinaldi F, Mattisi I et al (2010) Widespread cortical thinning characterizes patients with MS with mild cognitive impairment. Neurology 74:321-328

Sailer M, Fischl B, Salat D et al (2003) Focal thinning of the cerebral cortex in multiple sclerosis. Brain 126:1734-1744

Singh V, Chertkow H, Lerch JP, Evans AC, Dorr AE, Kabani NJ (2006) Spatial patterns of cortical thinning in mild cognitive impairment and Alzheimer's disease. Brain 129:2885-2893 Calabrese M, Oh MS, Favaretto A et al (2012) No MRI evidence of cortical lesions in neuromyelitis optica. Neurology 79:1671-1676 Chiaravalloti ND, DeLuca J (2008) Cognitive impairment in multiple sclerosis. Lancet Neurol 7:1139-1151

Polman CH, Reingold SC, Edan G et al (2005) Diagnostic criteria for multiple sclerosis: 2005 revisions to the "McDonald Criteria". Ann Neurol 58:840-846

Sled JG, Zijdenbos AP, Evans AC (1998) A nonparametric method for automatic correction of intensity nonuniformity in MRI data. IEEE Trans Med Imaging 17:87-97 for clinical trials: application to multiple sclerosis. IEEE Trans Med Imaging 21:1280-1291 outer surfaces of cerebral cortex from MRI. Neuroimage 12:340-356

Lerch JP, Evans AC (2005) Cortical thickness analysis examined through power analysis and a population simulation. Neuroimage 24:163-173

Chung MK, Worsley KJ, Robbins S et al (2003) Deformation-based surface morphometry applied to gray matter deformation. Neuroimage 18:198-213

Kabani N, Le Goualher G, MacDonald D, Evans AC (2001) Measurement of cortical thickness using an automated 3-D algorithm: a validation study. Neuroimage 13:375-380 Charil A, Dagher A, Lerch JP, Zijdenbos AP, Worsley KJ, Evans AC (2007) Focal cortical atrophy in multiple sclerosis: relation to lesion load and disability. Neuroimage 34:509-517 Querbes O, Aubry F, Pariente J et al (2009) Early diagnosis of Alzheimer's disease using cortical thickness: impact of cognitive reserve. Brain 132:2036-2047

Voineskos AN, Foussias G, Lerch J et al (2013) Neuroimaging evidence for the deficit subtype of schizophrenia. JAMA Psychiatry 70:472-480

von Glehn F, Jarius S, Cavalcanti Lira RP et al (2014) Structural brain abnormalities are related to retinal nerve fiber layer thinning and disease duration in neuromyelitis optica spectrum disorders. Mult Scler Jan 29. [Epub ahead of print]

Ceccarelli A, Rocca MA, Pagani E et al (2008) A voxel-based morphometry study of grey matter loss in MS patients with different clinical phenotypes. Neuroimage 42:315-322 in neuromyelitis optica. Ann Neurol 73:65-76

Rueda Lopes FC, Doring T, Martins C et al (2012) The role of demyelination in neuromyelitis 
optica damage: diffusion-tensor MR imaging study. Radiology 263:235-242

36 Suzuki M, Obara K, Sasaki Y et al (2003) Comparison of perivascular astrocytic structure between white matter and gray matter of rats. Brain Res 992:294-297

37 Rossi A, Crane JM, Verkman AS (2011) Aquaporin-4 Mz isoform: brain expression, supramolecular assembly and neuromyelitis optica antibody binding. Glia 59:1056-1063

38 Lucchinetti CF, Popescu BF, Bunyan RF et al (2011) Inflammatory cortical demyelination in early multiple sclerosis. N Engl J Med 365:2188-2197

39 Filippi M, Rocca MA, Benedict RH et al (2010) The contribution of MRI in assessing cognitive impairment in multiple sclerosis. Neurology 75:2121-2128

40 Hulst HE, Steenwijk MD, Versteeg A et al (2013) Cognitive impairment in MS: impact of white matter integrity, gray matter volume, and lesions. Neurology 80:1025-1032 
Table 1. Demographic and clinical characteristics ${ }^{a}$

\begin{tabular}{lllll}
\hline & MS & NMO & HC & p-value \\
\hline $\mathbf{N}$ & 27 & 23 & 26 & $/$ \\
Gender & $8 \mathrm{M} / 19 \mathrm{~F}$ & $3 \mathrm{M} / 20 \mathrm{~F}$ & $9 \mathrm{M} / 17 \mathrm{~F}$ & $0.21^{\mathrm{b}}$ \\
Age (years) & $33.15 \pm 9.40$ & $35.48 \pm 10.75$ & $35.08 \pm 11.28$ & $0.70^{\mathrm{c}}$ \\
& $(11-49)$ & $(17-56)$ & $(21-57)$ & \\
Education (years) & $13.96 \pm 2.70$ & $13.43 \pm 2.89$ & $13.85 \pm 3.29$ & $0.81^{\mathrm{c}}$ \\
& $(9-19)$ & $(9-19)$ & $(9-19)$ & \\
EDSS & $3.33 \pm 1.45$ & $4.52 \pm 1.76$ & $/$ & $0.01^{\mathrm{d}}$ \\
& $(0-6.5)$ & $(1-8)$ & & \\
PASAT3 & $42.00 \pm 9.82$ & $43.62 \pm 7.40$ & $53.65 \pm 6.58$ & $<0.01^{\mathrm{c}}$ \\
& $(29-57)^{\mathrm{e}}$ & $(26-54)^{\mathrm{e}}$ & $(29-59)$ & \\
PASAT2 & $35.94 \pm 10.12$ & $33.46 \pm 8.22$ & $47.35 \pm 8.63$ & $<0.01^{\mathrm{c}}$ \\
& $(21-58)^{\mathrm{e}}$ & $(19-48)^{\mathrm{e}}$ & $(22-57)$ & \\
MMSE & $26.60 \pm 1.51$ & $26.80 \pm 1.75$ & $29.87 \pm 0.34$ & $<0.01^{\mathrm{c}}$ \\
& $(25-30)^{\mathrm{e}}$ & $(23-29)^{\mathrm{e}}$ & $(29-30)$ & \\
Disease Duration (years) & $42.53 \pm 29.48$ & $51.91 \pm 54.73$ & $/$ & $0.45^{\mathrm{d}}$ \\
& $(0.4-10)$ & $(0.2-17)$ & & \\
\hline
\end{tabular}

Abbreviations: $\mathrm{MS}=$ multiple sclerosis; $\mathrm{NMO}=$ neuromyelitis optica; $\mathrm{HC}=$ healthy control; EDSS = Expanded Disability Status Scale; PASAT3 = the Paced Auditory Serial Addition Test 3 seconds; PASAT2 $=$ the Paced Auditory Serial Addition Test 2 seconds; MMSE = Mini Mental State Examination .

Data are presented as mean \pm SD (range) except $N$ and Gender.

${ }^{\mathrm{b}}$ Chi-square test.

${ }^{c}$ Main effect of group in ANOVA.

d 2-sample t-test.

${ }^{\mathrm{e}} \mathrm{p}<0.01$ compared to $\mathrm{HC}$ in post-hoc t-test. 
Table 2. Comparisons of global, hemispheric and lobar CTh among MS, NMO and HC groups

\begin{tabular}{|c|c|c|c|c|c|c|c|}
\hline & MS & NMO & $\mathrm{HC}$ & F-test & MS-HC & MS-NMO & NMO-HC \\
\hline Global Mean & $.20 \pm 0.19$ & $.27 \pm 0.12$ & $3.32 \pm 0.10$ & $6.66(0.0022)$ & $-3.63(0.00054)$ & -2.03 (n.s.) & -1.41 (n.s.) \\
\hline phere & & & 1 & & 18) & .00 (n.s.) & (n.s.) \\
\hline Right $\mathrm{He}$ & & & & & & & 3 (n.s.) \\
\hline Left & 0 & & & & 345) & -1.44 (n.s.) & 5 (n.s.) \\
\hline Le & .22 & & & & & -1.25 (n.s.) & n.s.) \\
\hline & $3.40 \pm 0.23$ & & & & -3 & -2.42 (n.s.) & n.s.) \\
\hline & $2.88 \pm 0.20$ & 2.93 & 3.0 & & & -1.33 (n.s.) & (n.s.) \\
\hline Riç & $3.25 \pm 0.22$ & 3.25 & $3.34 \pm 0.13$ & 2.88 & -2.40 & -1.04 (n.s.) & (n.s.) \\
\hline Riç & $2.99 \pm 0.18$ & 3.06 & $3.09 \pm 0.09$ & 4.42 ( & -2.86 & -2.05 (n.s.) & 7 (n.s.) \\
\hline Riç & $3.41 \pm 0.23$ & $3.55 \pm 0.13$ & $3.57 \pm 0.13$ & $7.77(0$ & -3.61 & $-3.07(0.0031)^{\pi}$ & 3 (n.s.) \\
\hline Right Occipita & $3.03 \pm 0.17$ & $3.10 \pm 0.14$ & $3.16 \pm 0.12$ & $6.32(0.0030)$ & $-3.55(0.00069)$ & -1.74 (n.s.) & -1.63 (n.s.) \\
\hline
\end{tabular}

This table shows the mean and standard deviation of global and lobar CTh in each group. F-values and t-values were derived from ANCOVA of mean CTh of the whole cortex, both hemispheres and 8 lobes (4 in each hemisphere). The numbers in brackets were p-value, where n.s. means non-significant.

${ }^{*} \mathrm{p}<0.05$ after FDR correction. 
Table 3. AAL regions revealed significant CTh differences among MS, NMO and HC groups

\begin{tabular}{|c|c|c|c|c|c|c|c|c|}
\hline Lobe & AAL Region & MS & NMO & $\mathrm{HC}$ & F-test & MS-HC & MS-NMO & NMO-HC \\
\hline \multirow[t]{2}{*}{ Frontal Lobe } & $\begin{array}{l}\text { Left Inferior frontal gyrus, opercular } \\
\text { part }\end{array}$ & $3.32 \pm 0.30$ & $3.41 \pm 0.19$ & $3.52 \pm 0.10$ & $7.04(0.0016)$ & $-3.74(0.00038)^{* *}$ & -1.41 (n.s.) & -2.13 (n.s.) \\
\hline & $\begin{array}{l}\text { Left Inferior frontal gyrus, triangular } \\
\text { part }\end{array}$ & $3.51 \pm 0.23$ & $3.50 \pm 0.22$ & $3.62 \pm 0.19$ & $6.48(0.0026)$ & $-3.52(0.00076)^{\star *}$ & -0.96 (n.s.) & -2.36 (n.s.) \\
\hline \multirow{9}{*}{$\begin{array}{l}\text { Occipital } \\
\text { Lobe }\end{array}$} & Right Lingual gyrus & $3.15 \pm 0.27$ & $3.20 \pm 0.15$ & $3.25 \pm 0.16$ & $8.97(0.00034)$ & $-4.23(0.000069)^{\star *}$ & -2.01 (n.s.) & -2.01 (n.s.) \\
\hline & Left Calcarine & $3.21 \pm 0.16$ & $3.22 \pm 0.19$ & $3.28 \pm 0.18$ & $8.82(0.00038)$ & $-4.03(0.00014)^{\star *}$ & -0.80 (n.s.) & $-3.00(0.0037)^{\star *}$ \\
\hline & Left Inferior occipital gyrus & $3.21 \pm 0.19$ & $3.28 \pm 0.16$ & $3.32 \pm 0.12$ & $8.55(0.00048)$ & $-3.89(0.00023)^{* *}$ & $-3.05(0.0032)^{* *}$ & -0.65 (n.s.) \\
\hline & Left Fusiform gyrus & $3.26 \pm 0.21$ & $3.29 \pm 0.14$ & $3.36 \pm 0.11$ & $8.39(0.00054)$ & $-4.02(0.00014)^{* *}$ & -2.54 (n.s.) & -1.28 (n.s.) \\
\hline & Right Fusiform gyrus & $3.62 \pm 0.28$ & $3.66 \pm 0.19$ & $3.72 \pm 0.17$ & $6.75(0.0021)$ & $-3.67(0.00047)^{* *}$ & -1.77 (n.s.) & -1.71 (n.s.) \\
\hline & Left Lingual gyrus & $3.09 \pm 0.24$ & $3.14 \pm 0.17$ & $3.26 \pm 0.10$ & $6.56(0.0024)$ & $-3.62(0.00055)^{* *}$ & -1.71 (n.s.) & -1.72 (n.s.) \\
\hline & Right Inferior occipital gyrus & $3.45 \pm 0.32$ & $3.54 \pm 0.22$ & $3.60 \pm 0.18$ & $5.42(0.0065)$ & $-3.27(0.0017)^{* *}$ & -1.83 (n.s.) & -1.28 (n.s.) \\
\hline & Right Calcarine & $3.47 \pm 0.33$ & $3.53 \pm 0.22$ & $3.54 \pm 0.19$ & $5.08(0.0087)$ & $-3.09(0.0028)^{\star *}$ & -0.76 (n.s.) & -2.16 (n.s.) \\
\hline & Left Middle occipital gyrus & $3.39 \pm 0.24$ & $3.46 \pm 0.26$ & $3.51 \pm 0.27$ & $4.90(0.010)$ & $-3.03(0.0035)^{\star *}$ & -0.69 (n.s.) & -2.17 (n.s.) \\
\hline \multirow[t]{2}{*}{ Parietal Lobe } & Right Supramarginalgyrus & $3.09 \pm 0.25$ & $3.10 \pm 0.22$ & $3.13 \pm 0.24$ & $8.60(0.00046)$ & $-4.11(0.00011)^{* *}$ & -2.39 (n.s.) & -1.52 (n.s.) \\
\hline & Right Postcentralgyrus & $3.43 \pm 0.30$ & $3.51 \pm 0.17$ & $3.53 \pm 0.18$ & $5.36(0.0068)$ & $-3.06(0.0031)^{* *}$ & -2.44 (n.s.) & -0.47 (n.s.) \\
\hline \multirow{6}{*}{$\begin{array}{l}\text { Temporal } \\
\text { Lobe }\end{array}$} & Left Parahippocampalgyrus & $2.92 \pm 0.26$ & $2.97 \pm 0.21$ & $3.02 \pm 0.20$ & $15.40(0.0000029)$ & $-5.26(0.0000015)^{* *}$ & $-4.00(0.00016)^{* *}$ & -1.01 (n.s.) \\
\hline & Right Parahippocampalgyrus & $2.86 \pm 0.27$ & $2.97 \pm 0.20$ & $2.94 \pm 0.19$ & $12.25(0.000028)$ & $-4.94(0.0000051)^{* *}$ & -2.01 (n.s.) & -2.66 (n.s.) \\
\hline & Right Heschlgyrus & $3.26 \pm 0.20$ & $3.33 \pm 0.17$ & $3.35 \pm 0.14$ & $9.10(0.00031)$ & $-3.98(0.00017)^{* *}$ & $-3.21(0.0020)^{\star *}$ & -0.58 (n.s.) \\
\hline & Right Insula & $3.28 \pm 0.17$ & $3.30 \pm 0.19$ & $3.38 \pm 0.14$ & $8.33(0.00057)$ & $-3.32(0.0014)^{\star *}$ & $-3.64(0.00052)^{\star *}$ & 0.46 (n.s.) \\
\hline & Right Superior temporal gyrus & $3.03 \pm 0.35$ & $3.07 \pm 0.28$ & $3.15 \pm 0.23$ & $7.11(0.0015)$ & $-3.60(0.00059)^{* *}$ & -2.67 (n.s.) & -0.75 (n.s.) \\
\hline & Left Insula & $3.68 \pm 0.21$ & $3.67 \pm 0.23$ & $3.76 \pm 0.21$ & $5.82(0.0046)$ & $-3.30(0.0015)^{\star *}$ & -2.30 (n.s.) & -0.83 (n.s.) \\
\hline \multirow[t]{2}{*}{$\begin{array}{l}\text { Limbic } \\
\text { System }\end{array}$} & Right Anterior cingulate & $3.30 \pm 0.25$ & $3.37 \pm 0.23$ & $3.46 \pm 0.22$ & $6.20(0.0033)$ & $-3.50(0.00080)^{* *}$ & -1.31 (n.s.) & -2.01 (n.s.) \\
\hline & Left Anterior cingulate & $3.38 \pm 0.33$ & $3.45 \pm 0.19$ & $3.48 \pm 0.24$ & $4.95(0.0098)$ & $-3.06(0.0031)^{* *}$ & -2.06 (n.s.) & -0.85 (n.s.) \\
\hline
\end{tabular}

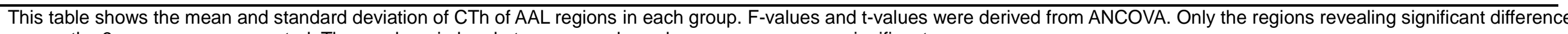
among the 3 groups were presented. The numbers in brackets were $p$-value, where n.s. means non-significant.
}

${ }^{* *} \mathrm{p}<0.05$ after FDR correction. 
Figure Legends:

Figure 1. The flowchart of CIVET software. The structural images were first corrected for non-uniformity and registered to stereotaxic space (1). Then the images were segmented (2) and the grey matter/white matter interface was extracted (3-4). The grey matter/CSF interface was sequentially determined by expanding the grey matter/white matter interface (5). Finally, the CTh was measured on each vertex and smoothed by a $20 \mathrm{~mm}$ surface-based kernel (6).

Figure 2. Mean cortical thickness (CTh) of the subjects in MS, NMO and HC groups. The mean CTh of the whole cortical (left), the left hemisphere (median) and the right hemisphere (right) were shown in the figure.

${ }^{* * *} p<0.001$

${ }^{*} \mathrm{p}<0.05$

Figure 3. Vertex-based CTh ANCOVA results after FDR correction $(p<0.05)$. (A) F-value map depicting main effect of groups. (B) T-value map of post-hoc comparison between MS and HC. (C) T-value map of post-hoc comparison between MS and NMO. (D) T-value map of post-hoc comparison between $\mathrm{NMO}$ and $\mathrm{HC}$. Age and gender were taken as covariates in ANCOVA.

Figure 4. Correlations between vertex-based CTh and clinical variables including PASAT2, PASAT3 and disease duration in patients with MS. 


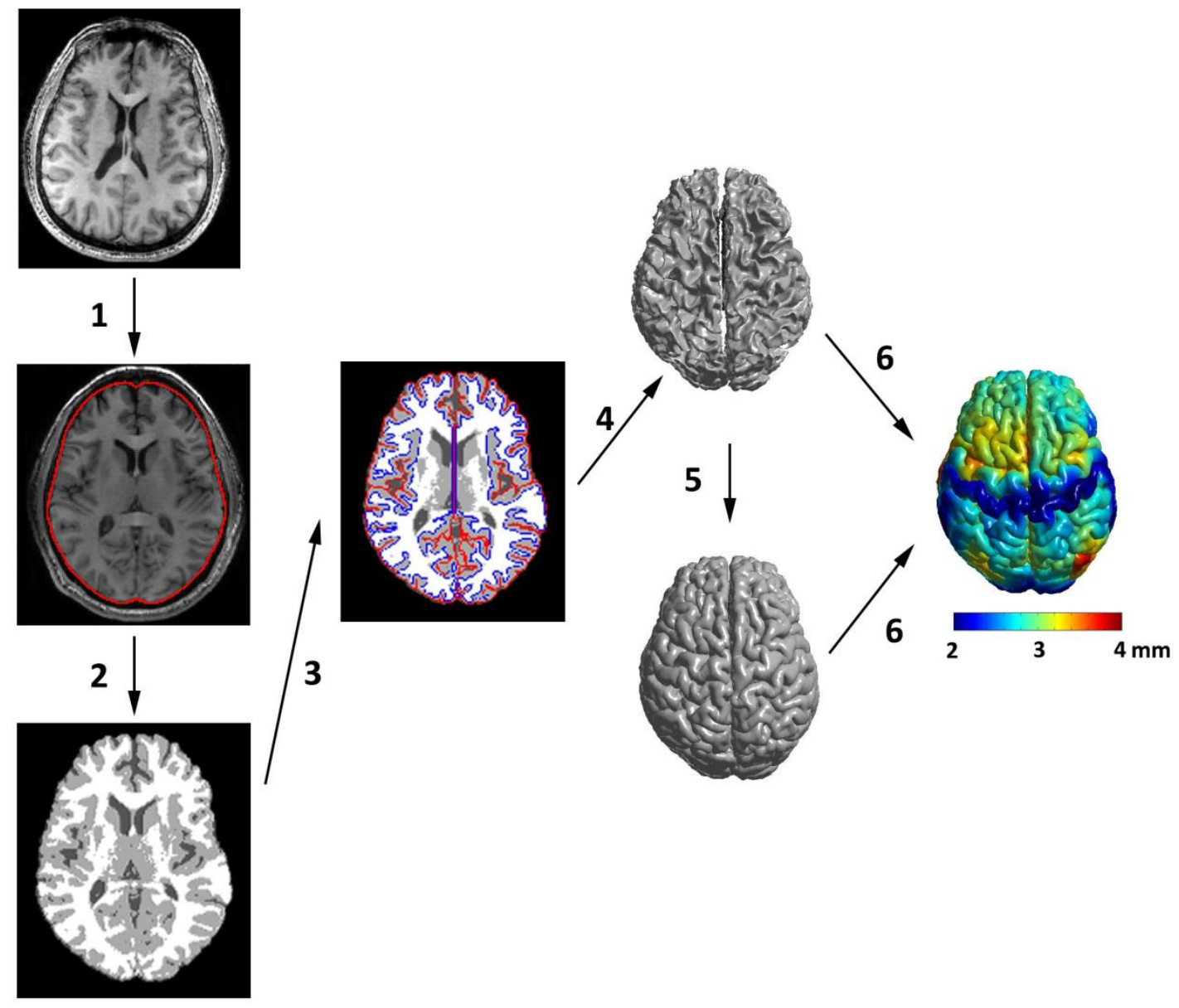



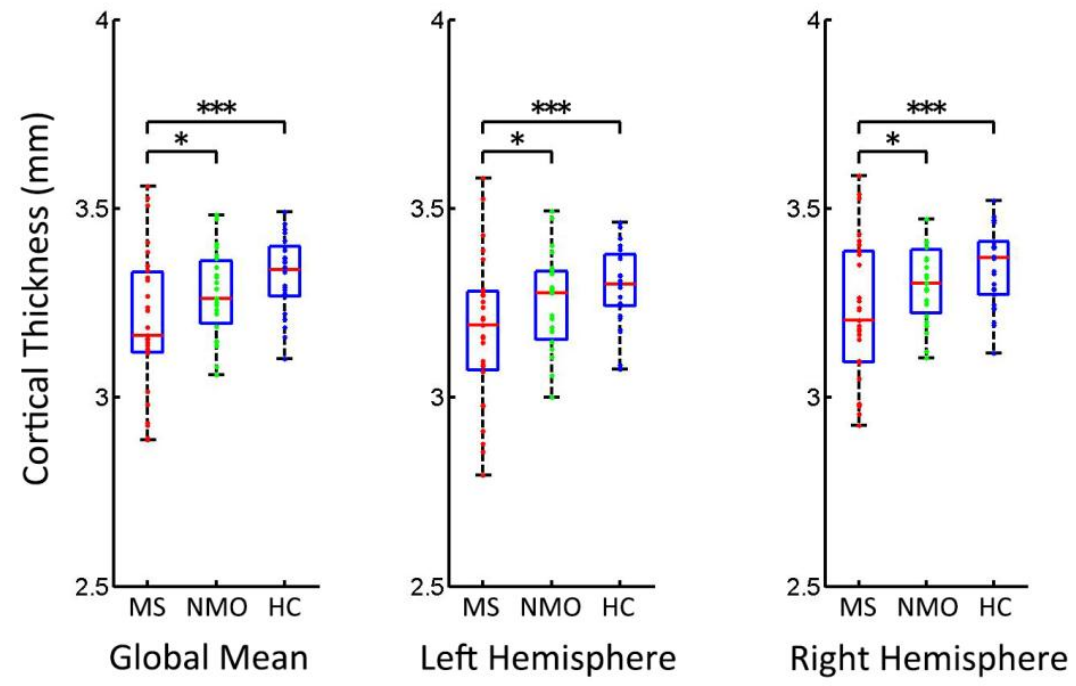
A

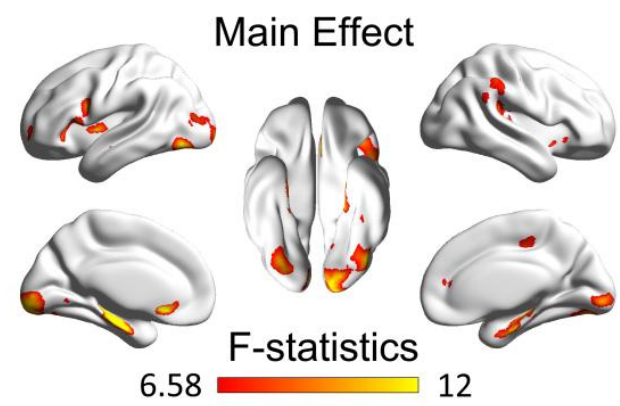

C

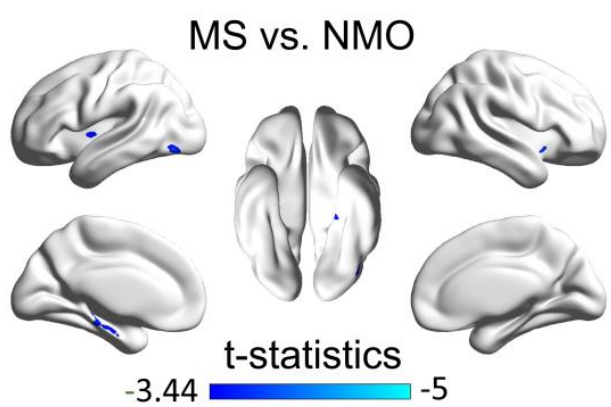

B

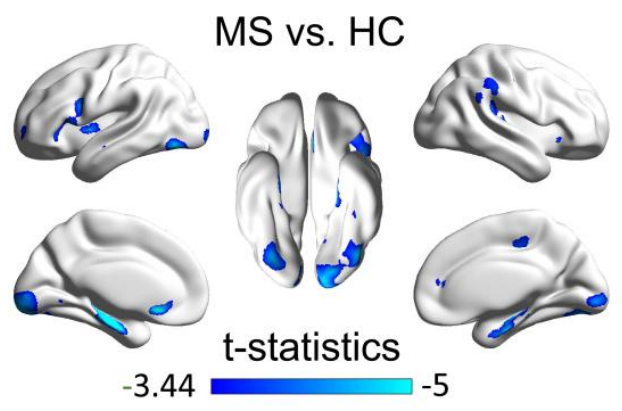

D

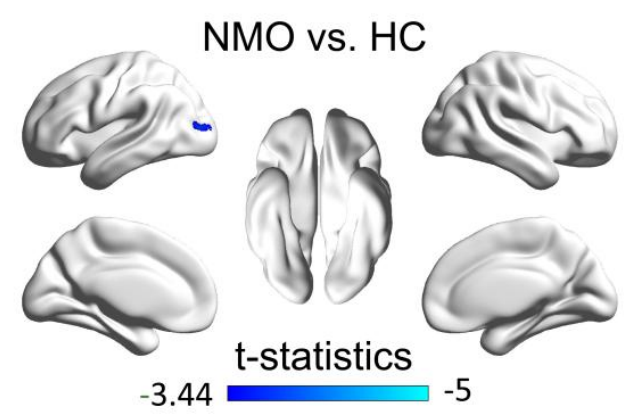




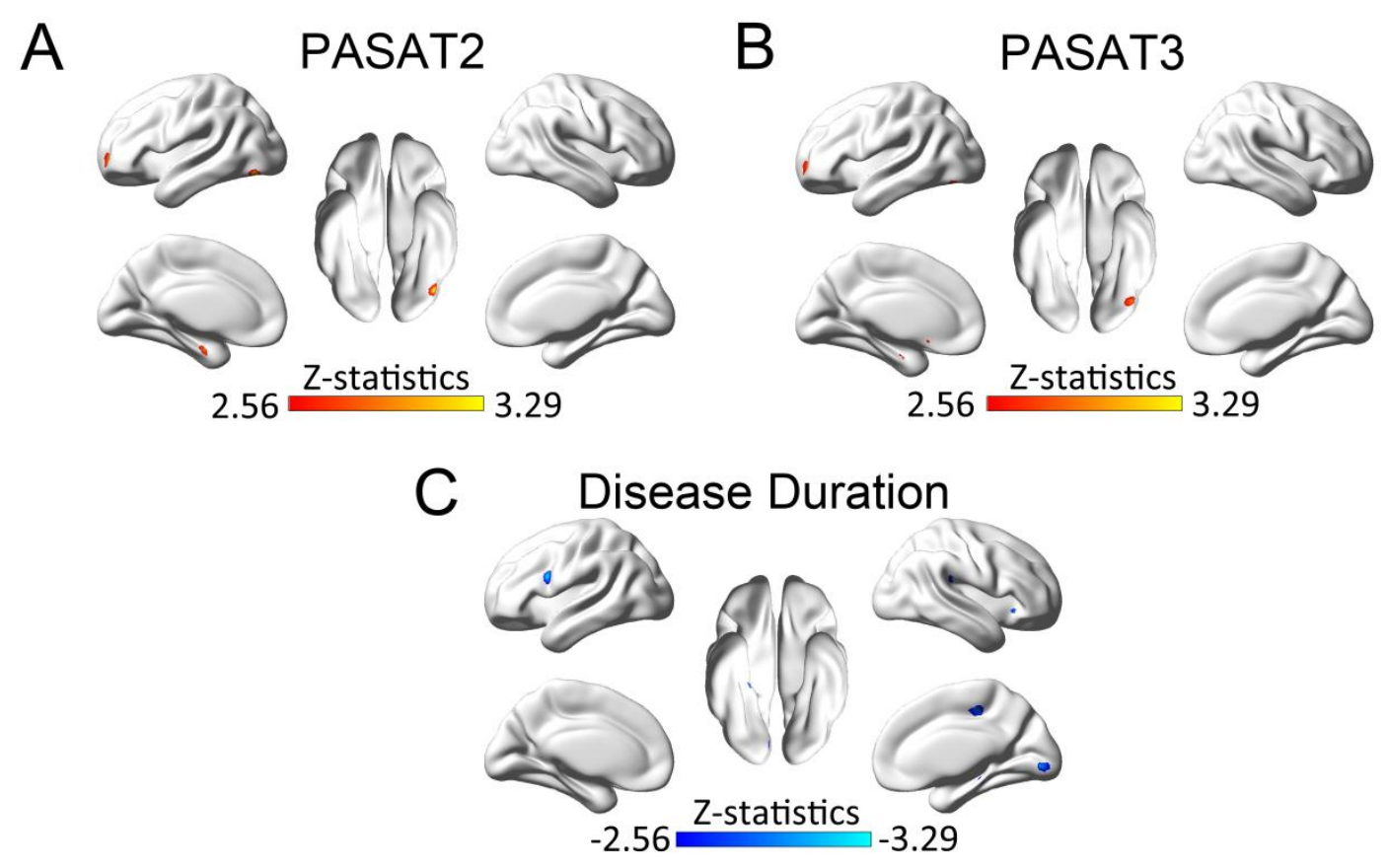

